\title{
ON THE EDGE-BALANCE INDEX SETS OF $L$-PRODUCT OF CYCLES
}

\author{
Daniel Bouchard ${ }^{1}$, Patrick Clark ${ }^{2}$, and Hsin-Hao $\mathrm{Su}^{3}$ \\ ${ }^{1}$ Department of Mathematics, Stonehill College, \\ Easton, MA 02357, USA, dbouchard@students.stonehill.edu \\ ${ }^{2}$ Department of Mathematics, Stonehill College, \\ Easton, MA 02357, USA, pclark1@students.stonehill.edu \\ ${ }^{3}$ Department of Mathematics, Stonehill College, \\ Easton, MA 02357, USA, hsu@stonehill.edu
}

\begin{abstract}
Let $G$ be a simple graph with vertex set $V(G)$ and edge set $E(G)$, and let $\mathbb{Z}_{2}=\{0,1\}$. Any edge labeling $f$ induces a partial vertex labeling $f^{+}$: $V(G) \rightarrow \mathbb{Z}_{2}$ assigning 0 or 1 to $f^{+}(v), v$ being an element of $V(G)$, depending on whether there are more 0-edges or 1-edges incident with $v$, and no label is given to $f^{+}(v)$ otherwise. For each $i \in \mathbb{Z}_{2}$, let $v_{f}(i)=\left|\left\{v \in V(G): f^{+}(v)=i\right\}\right|$ and let $e_{f}(i)=|\{e \in E(G): f(e)=i\}|$. An edge-labeling $f$ of $G$ is said to be edge-friendly if $\left\{\left|e_{f}(0)-e_{f}(1)\right| \leq 1\right\}$. The edge-balance index set of the graph $\mathrm{G}$ is defined as $E B I(G)=\left\{\left|v_{f}(0)-v_{f}(1)\right|: f\right.$ is edge-friendly. $\}$. In this paper, exact values of the edge-balance index sets of $L$-product of cycles with cycles, $C_{n} \times{ }_{L} C_{m}$ are presented.

Key words: Edge labeling, edge-friendly labeling, cordiality, edge-balance index set, $L$-products, cycles.
\end{abstract}

2000 Mathematics Subject Classification: 05C78, 05C25.

Received: 09-08-2011, revised: 09-09-2011, accepted: 04-12-2012. 